\title{
Human Papillomavirus (HPV) Type Distribution in Females with Abnormal Cervical Cytology. A Correlation with Histological Study
}

\author{
Fernando Cobo ${ }^{*}, 1$, Ángel Concha ${ }^{1}$ and Marta Ortiz ${ }^{2}$ \\ ${ }^{I}$ Infectious Pathology Unit, Department of Pathology, Hospital Universitario Virgen de las Nieves, Granada, Spain \\ ${ }^{2}$ Centro Nacional de Microbiología, Madrid, Spain
}

\begin{abstract}
The aim of this study was to determine human papillomavirus (HPV) types distribution in cervical preneoplasic lesions in a Southern Spanish population and their relationship between HPV type and grade of histopathological abnormality. Finally, 232 cervical samples from 135 women with previous cytological abnormalities were included in this study. Colposcopy studies and biopsies were performed. Haematoxylin-eosin stained slides were observed and detection of HPV DNA in cervical swabs was carried out with use of a polymerase chain reaction and microarrays technology. The relationship between the presence of HPV infection and diagnostic variables was evaluated. HPV 16 was the most common type followed by HPV 58, 51, 33 and 31. However, the two HPV types targeted in the prophylactic vaccines such as HPV type 16 and 18 were detected in only $37(21.2 \%)$ and $2(1.1 \%)$ cases respectively. Thirty-three (18.9\%) of samples were infected with multiple types, the majority of them with two types. In addition, during the follow-up of patients many changes in type distribution were observed. Several studies will be necessary in order to evaluate the HPV type distribution for therapeutically and prophylactic purposes such as vaccine treatment. Also, because of the differences obtained depending of use of various DNA technologies, the performance of some comparative studies of the different methods from detection of HPV would be advisable in a high population of patients and with the most homogeneous conditions possible.
\end{abstract}

\section{INTRODUCTION}

Human papillomavirus (HPV) is small, icosahedral and non-enveloped viruse now grouped in the papillomaviridae family. The HPV genome consists of a single molecule of double-stranded circular DNA within a capsid composed of two structural proteins (L1 and L2 proteins) [1].

At the moment, more than 100 types of HPV have been identified and these correspond to 16 different genus; their classification is now based on the nucleotide sequences comparison of specific regions such as coding regions of E6, E7 and L1 proteins [2]. Depending of this, the types have less than $90 \%$ of homology in the sequences of such regions. The clinically most important genus is the $\alpha$-papillomavirus which contain all HPV associated with mucosal lesions. $\beta$ papillomaviruses include all the kind of virus associated with epidermodysplasia verruciformis.

HPV is one of the most common causes of sexually transmitted diseases worldwide. The current prevalence of HPV infection in women range to $2-44 \%$, being Asia and Europe where it can found the less percentage of infection [3].

HPV is associated with a wide variety of clinical conditions and diseases that range from innocuous lesions to certain neoplasias. Firstly, HPV was recognized as the cause of cutaneous warts, although this infection is also related

*Address correspondence to this author at the Infectious Pathology Unit, Department of Pathology, Hospital Universitario Virgen de las Nieves, Avenida Fuerzas Armadas, 2, 18014 Granada, Spain; Tel: 9580206 91; Fax: 9580201 32; E-mail: fernando.cobo.sspa@juntadeandalucia.es to focal epithelial hyperplasia of the oral cavity (Heck's disease), epidermodysplasia verruciformis, recurrent respiratory papillomatosis and conjunctival papillomas and carcinomas [4]. Moreover, several epidemiological studies permit to conclude that certain HPV type infections are a necessary factor for the development to cervical cancer [5-7]. These kind of neoplasias represent the second most common cancer in women worldwide and contribute with approximately $10 \%$ of overall female cancers. Other tumours HPV-related are anal, vaginal, vulvar, penile, laryngeal and oropharyngeal cancer, so HPV is estimated responsible to $5 \%$ of all kind of neoplasias worldwide [8]. In fact, in 1990 was described the presence of HPV type 16 in laryngeal carcinomas [9].

From an epidemiological point of view and based on their association with cervical cancer and precursor lesions, $\mathrm{HPV}_{\mathrm{s}}$ have been classified in both high-risk and low-risk HPV types; now, there are also two additional groups: probably carcinogenic high-risk and non-determinate risk of carcinogenesis [10].

The commonest HPV infection diagnostic methods in cervical pathology have been the cytology (both conventional and monolayer cytology) by means of Papanicolaou-stained smear (Pap smear) [11] and histology, in which patients with abnormal cytology are usually evaluated by colposcopy and biopsy. Molecular methods to detect HPV DNA sequences (include the possibility of HPV typing) have been recently introduced in order to improve this diagnosis.

Recently, both the Federal Drug Administration (FDA) and the European Agency for the Evaluation of Medicinal 
Products (EMEA) have approved and regulated the use of two HPV vaccines (Gardasil ${ }^{\circledR}$ and Cervarix ${ }^{\circledR}$ ) [12]. In phase II/III studies, both vaccines have demonstrated safety and efficacy to the prevention of HPV infections caused by the viral types including in these.

The main objective of this study was to perform the diagnosis of HPV infection in a group of female patients with cytological abnormalities referred to the Cervix Cancer Diagnosis Unit (CCDU) of our hospital by means of histopathological and molecular techniques; additionally, a correlation between both methods was also carried out. Moreover, a second objective was to stablish a relationship between HPV type and grade of histopathological abnormality.

\section{MATERIALS AND METHODOLOGY}

\section{Study Design and Population}

The patients included in this study were first-visited from February 2006 to February 2007; a population-based cohort of women with cytological abnormalities visualized by means of Pap smears (presence of non-reactive atypical cells) was used in this study. The total number of Pap smears performed during the study period by the Early Cervical Cancer Detection Program (ECCDP) was 45,000, approximately. The population came from a health area of approximately 450,000 inhabitants corresponding to the North of the province of Granada (Andalucía, Spain). From these, 250,000 are eligible women for participation in the ECCDP, but only 1,500 women were send from the health basic centres to the CCDU of our Hospital to complete the study. Finally, 232 samples for HPV typing and 173 biopsies corresponding to 135 women were included in this report. The main inclusion criterion was the presence of cytological alterations in the Pap smears and further remission to our hospital in order to complete the study. The only exclusion criteria were women who refusing to continue in follow up. The follow-up visits were established each 6 months during 3 years. The collected data were the patient age, HPV types and biopsy sample diagnosis. This histological diagnosis was classified in five categories as follows: inflammatory/reactive lesions, undetermined/not conclusive lesions such as atypia borderline, LSIL (low-grade squamous intraepithelial lesions), HSIL (high-grade squamous intraepithelial lesions), and invasive carcinoma.

\section{Collection of Data and Specimens}

The patients were studied and treated according the protocol of the Department of Gynaecology and Obstetric of our Hospital. The patients finally included in the study signed an inform consent approved by the ethical committee of our Hospital. In the first and follow-up visits at the hospital, the women were interviewed by a trained physician in order to know information about risk factors for cervical cancer. Furthermore, a gynaecologist performed a pelvic examination including a colposcopy study and, it was necessary, a biopsy sample was taken. Biopsy specimens were formalin fixed and further paraffin embedded (Histosec $^{\circledR}$, Merck, Darmstadt, Germany) by means of manufacture's recommendation; later, a pathological report was then discharged. Simultaneously, a cervical swab without transport medium was collected for HPV DNA detection and typing study.

\section{HPV Detection and Typing}

Detection of HPV DNA in cervical swabs was carried out by means of a polymerase chain reaction (PCR)-based assay and further typing by the kit Clinical Arrays ${ }^{\circledR}$ (Genómica, Madrid, Spain) [13]. The detection limit of this technique is 10 copies of DNA virus, without any difference among HPV types. Briefly, the samples were processed as follows: the DNA extraction, purification and amplification was carried out by means of this kit, amplifying a DNA-target of $450 \mathrm{bp}$ inside L1 protein. A 9-min initial denaturation step at $95^{\circ} \mathrm{C}$, 45 cycles of $1 \mathrm{~min}$ at $94^{\circ} \mathrm{C}, 1 \mathrm{~min}$ at $55^{\circ} \mathrm{C}, 1 \mathrm{~min}$ at $72^{\circ} \mathrm{C}$, and a chain final elongation step at $72^{\circ} \mathrm{C}$ for $9 \mathrm{~min}$. The HPV typing was then performed by means a new technological platform based in low density microarrays hybridization assay (Clinical Arrays ${ }^{\circledR}$ genotyping), in which the microchip is inserted at the bottom of a Eppendorf tube. The presence of the 35 most important HPV genotypes could be analyzed by means of this method. The extracted DNA remain frozen and stored in the Tumour and Tissue Bank at our hospital.

Twenty-three DNA samples were send to the National Centre of Microbiology, the reference centre in Spain, in order to carry out an external quality control. This establishment used a Real-Time PCR method (Roche Linear Array) for the HPV diagnosis and typing [14]. These samples were selected on basis to peculiar results such as the presence of numerous (multiple infections) or infrequent (uncommon) HPV types.

\section{Statistical Analysis}

The statistical software package SPSS 13.0 for Windows was used for this analysis. A descriptive analysis of the variables was firstly carried out; the percentages were calculated for the qualitative variables and means and standard deviations for the numerical variable.

Later, the relationship between the dependent variable (HPV presence or not) and the independent variables (age and diagnostic variables) was performed by means of the chi-square test, and we consider a significant value $\mathrm{p}<0.01$.

The sample size (number of patients included in the study of 135) was calculated in basis to an error of $4 \%$.

\section{RESULTS}

\section{Study Subjects}

Of the 135 women who were included in this study, 72 $(53 \%)$ were 34 years old or younger, 38 (28\%) were between 35 and 44 years old and 25 (18\%) were 45 years old or older. The mean age at enrolment was 35 years (standard deviation $=10.4$ years).

A total of 232 samples for HPV typing corresponding to 135 women were consecutively included in this study. In addition, 173 biopsies were taken. The histological diagnosis was: 67 with inflammatory/reactive non specific lesions, 68 with LSIL, 24 with HSIL and 14 with atypia borderline; in the remaining samples, the histological study was not performed due to a gynaecologist criteria. All the samples were simultaneously tested for HPV DNA presence. 


\section{Specific Prevalence of HPV Genotypes}

A total of 174 samples $(75 \%)$ were positive for HPV DNA and $55(23.7 \%)$ were negative. In three samples, DNA was not obtained (Table 1).

Table 1. Human Papillomavirus (HPV) Type Distribution in Cervical Samples Detected by PCR-Microarrays in Granada, Spain

\begin{tabular}{|l|l|}
\hline \multicolumn{1}{|c|}{ HPV Detection } & \multicolumn{1}{|c|}{$\mathbf{N}^{\mathbf{0}} \mathbf{( \% )}^{*}$} \\
\hline \hline Positive & 174 \\
Negative & 55 \\
DNA no detected & 3 \\
Single infections & $141(81.03 \%)$ \\
Multiple infections & $33(18.9 \%)$ \\
2 types & 24 \\
> 2 types & 9 \\
Type specific & \\
HPV 16 & $37(21.2 \%)$ \\
HPV 53 & $16(9.1 \%)$ \\
HPV 6 & $16(9.1 \%)$ \\
HPV 66 & $12(6.8 \%)$ \\
HPV 58 & $11(6.3 \%)$ \\
HPV 61 & $9(5.1 \%)$ \\
HPV 51 & $8(4.5 \%)$ \\
HPV 33 & $7(4.02 \%)$ \\
HPV 31 & $5(2.8 \%)$ \\
HPV 11 & $5(2.8 \%)$ \\
HPV 72 & $5(2.8 \%)$ \\
HPV 18 & $2(1.1 \%)$ \\
HPV 62 & $2(1.1 \%)$ \\
HPV 81 & $2(1.1 \%)$ \\
HPV 70 & $2(1.1 \%)$ \\
HPV 56 & $1(0.5 \%)$ \\
HPV 84 & $1(0.5 \%)$ \\
\hline &
\end{tabular}

N (\%): from the positive cases ( $\mathrm{n}=174)$.

From positive cases $(\mathrm{n}=174), 141(81.03 \%)$ were infected with a single HPV type, and $33(18.9 \%)$ were infected with multiple types. When the infection was caused by an only type, high-risk types supposed 73 samples (51.7\%). HPV 16 was the most common type (37 samples/21.2\%), followed by HPV type 58 (11 samples/6.3\%). The infection with low-risk types was found in 39 samples $(27.6 \%)$, and the type 6 was the most common infection in this group (16 samples/9.1\%). Finally, infection with probable high-risk types was obtained in 28 samples (16.09\%) (caused with HPV type 53 and 66). Only one case was caused with an unclassified HPV type.

With respect to the group of infections with multiple types, the majority of them were caused with 2 types (24 samples $/ 72.7 \%$ ). Three or more types was only obtained in 9 $(27.2 \%)$ of total cases. In these co-infections, 19 (57\%) had only high-risk types and 11 (33\%) had both high and low-risk types. One case had co-infection by not classified HPV types (HPV 83,84 ), and also one case had co-infection by a low-risk HPV type and probable high-risk HPV type. Curiously, the most common type involved in the co-infections was HPV 51 (12 samples/36\%), followed by HPV 16 (10 samples/30\%) (Table 2 ).
Taking into account overall data, HPV type 16 was the most common virus (47 samples/27.01\%), followed by HPV type $51 \quad(20 \quad$ samples/11.4\%) and type $58 \quad(19$ samples/10.9\%).

Table 2. Multiple HPV Infections in the Southern Spanish Health Area. Granada, Spain

\begin{tabular}{|c|c|}
\hline Patient & HPV Types \\
\hline 3 & 6,16 \\
\hline 9 & $6,16,18,33,58$ \\
\hline 11 & 6,54 \\
\hline 19 & $31,51,84$ \\
\hline 28 & 16,70 \\
\hline 35 & $11,58,66$ \\
\hline 62 & 56,61 \\
\hline 65 & 31,81 \\
\hline 70 & 31,62 \\
\hline 72 & 58,66 \\
\hline 75 & 33,58 \\
\hline 76 & 16,51 \\
\hline 77 & 83,84 \\
\hline 81 & 11,66 \\
\hline 82 & 6,16 \\
\hline 87 & $16,66,70$ \\
\hline 93 & 51,58 \\
\hline 93 & 51,58 \\
\hline 98 & $18,31,51$ \\
\hline 99 & 6,16 \\
\hline 100 & 16,53 \\
\hline 106 & $6,16,18,31,53$ \\
\hline 107 & 51,52 \\
\hline 108 & $16,33,58$ \\
\hline 116 & 51,66 \\
\hline 118 & 16,51 \\
\hline 120 & 51,53 \\
\hline 120 & 51,53 \\
\hline 121 & $33,52,53$ \\
\hline 127 & 6,51 \\
\hline 130 & 53,58 \\
\hline 131 & 52,56 \\
\hline 133 & $51,53,66$ \\
\hline
\end{tabular}

\section{External Quality Control}

In order to assure an adequate quality control of this analysis, 23 samples were selected and sent to our National Reference Centre (National Centre for Microbiology, 
Table 3. Correlation Between our Results and the External Quality Control (National Centre for Microbiology, Madrid, Spain) in 23 Samples with Multiple Infections

\begin{tabular}{|c|c|c|}
\hline Patient & Types Obtained with our Method (Microarrays) & Types Obtained in the External Control \\
\hline 3 & 6,16 & 16 \\
\hline 28 & 16,70 & $16,70+\mathbf{4 5 ,} 62$ \\
\hline 62 & 56,61 & $56,61+73$ \\
\hline 70 & 31,62 & 31,62 \\
\hline 75 & 33,58 & $33,58+42$ \\
\hline 76 & 16,51 & $16,51+35,45$ \\
\hline 77 & 83,84 & 83,84 \\
\hline 100 & 16,53 & $16,53+62,67$ \\
\hline 106 & $6,16,18,31,53$ & $6,16,18,31,53+\mathbf{5 2}$ \\
\hline 107 & $51, \mathbf{5 2}$ & 51 \\
\hline 108 & $16,33,58$ & $16,33,58+\mathbf{5 1}, \mathbf{I S 3 9}$ \\
\hline 116 & 51,66 & $51,66+\mathbf{1 6}, \mathbf{3 5}, \mathbf{4 0}, \mathbf{6 1}, \mathbf{8 1}, \mathbf{8 4}$ \\
\hline 118 & 16,51 & 51, CP6108 \\
\hline 120 & 51,53 & 51,53 \\
\hline 121 & $33, \mathbf{5 2}, 53$ & 33,53 \\
\hline
\end{tabular}

* Note the differences between both methods highlighted in bold.

Instituto de Salud Carlos III, Madrid). In general, the control was satisfactory. In all cases, both institutions detected the same HPV types, but in several samples, both institutions detected additional HPV types. Only in four cases, there were additional HPV types only obtained by our method; also, in 4 cases both, our laboratory and the National Reference Centre, obtained the same results. In one case, in which we have not found DNA, the external centre observed 3 HPV types. In Table $\mathbf{3}$, these results can be observed.

\section{Evolution of HPV Infection}

Two o more HPV analysis was carried out in 74 women. Independently of HPV type or histological diagnosis, in 40 cases (54\%) HPV infection disappeared during the study period. In 17 women (23\%), HPV types were the same in all analysis. In 5 women $(6 \%)$ additional HPV types could be observed in further analysis, and in $4(5 \%)$ a decrease of HPV types could be found. Only in two women with lowrisk HPV types were changed to high-risk HPV types. Finally high-risk HPV infection in 4 patients disappeared and later low-risk HPV types were found. In Table $\mathbf{4}$, it can see these results.

\section{Risk Associated with HPV}

With respect to relationship between the patients' age and the HPV presence or not, statistical significant differences in the age ranges $(\leq 34,35-44,>44$ years old $)$ were not observed (chi square, $\mathrm{p}=0.157$ ).

Also, a correlation between the presence of HPV in each group of histological diagnosis was carried out. The statistical analysis of this study was not relevant in our samples (Table 5). Briefly, in the LSIL and HSIL groups a higher number of high-risk HPV types than in the inflammatory lesions and ASCUS groups were observed; however, these differences were not statistical significant.

\section{DISCUSSION}

Two main stages could be consider in HPV epidemiology: type distribution of cervical HPV infection in women with normal cytology and in women with abnormal cytology. Recently, a meta-analysis of relevant studies about the worldwide prevalence and type distribution of cervical HPV DNA in women with normal cytology has been published [3]. In this manuscript, the overall HPV prevalence was estimated to be $10.4 \%$. However, there were some differences depending of 
Table 4. Evolution of HPV Infection in Women with Two or More HPV Analysis. Granada, Spain”

\begin{tabular}{|c|c|c|c|c|c|}
\hline Patient & First Sample & Second Sample & Third Sample & Fourth Sample & Evolution \\
\hline 6 & HPV 18 & HPV 66 & & & HR - probable HR \\
\hline 11 & HPV 66 & HPV 16 & HPV 6 & HPV 6, 54 & Probable HR - HR - LR \\
\hline 18 & HPV 58 & HPV 51 & HPV 11 & & $\mathrm{HR}-\mathrm{HR}-\mathrm{LR}$ \\
\hline 19 & HPV 31 & HPV $31,51,84$ & & & $\mathrm{HR}-\mathrm{HR}+$ undetermined \\
\hline 27 & HPV 53 & HPV 11 & & & Probable HR - LR \\
\hline 35 & HPV 11 & HPV $11,58,66$ & & & $\mathrm{LR}-\mathrm{LR}+\mathrm{HR}+$ probable HR \\
\hline 46 & HPV 16 & HPV 6 & & & $\mathrm{HR}-\mathrm{LR}$ \\
\hline 72 & HPV 58, 66 & HPV 58 & & & $\mathrm{HR}+$ probable $\mathrm{HR}-\mathrm{HR}$ \\
\hline 75 & HPV 33,58 & HPV 33 & & & $\mathrm{HR}-\mathrm{HR}$ \\
\hline 81 & HPV 33 & HPV 11, 66 & & & $\mathrm{HR}-\mathrm{LR}+$ probable $\mathrm{HR}$ \\
\hline 82 & HPV 6, 16 & HPV 51 & & & $\mathrm{LR}+\mathrm{HR}-\mathrm{HR}$ \\
\hline 104 & HPV 6 & HPV 56 & & & $\mathrm{LR}-\mathrm{HR}$ \\
\hline 123 & HPV 58 & HPV 6 & & & $\mathrm{HR}-\mathrm{LR}$ \\
\hline 127 & HPV 51 & HPV 6, 51 & & & $\mathrm{HR}-\mathrm{LR}+\mathrm{HR}$ \\
\hline 133 & HPV $51,53,66$ & HPV 66 & & & $\mathrm{HR}+$ probable $\mathrm{HR}$ - probable $\mathrm{HR}$ \\
\hline
\end{tabular}

* In 40 women, HPV DNA disappeared in further analysis; in 17 patients, HPV type was the same in all samples.

HR: High-risk cancer; LR: Low-risk cancer.

the region of origin. Africa and Central America and Mexico were the regions where it found a higher prevalence of HPV infection, while Europe and Asia had less infection prevalence. In Europe a north/south pattern could be observed, with HPV prevalence decreasing with decreasing latitude [3]. In this study, HPV16 is the most common HPV type and the five most common HPV types in HPV-positive women worldwide were HPV16, HPV18, HPV31, HPV58 and HPV52, being 50\% of all HPV infections [3].

Other studies focused with the distribution of HPV types in women with cervical lesions. In a recent report, HPV 16 and 18 were the most frequent HPV types identified in invasive cancers $(80 \%)$, but the distribution patterns of HPV types in the intraepithelial lesions was highly varied [15].

In Spain, there are a few reports on HPV epidemiology. In 2006 a study performed in Alicante and Madrid was published, revealing that the prevalence of HPV infection among general population was $10.7 \%$ [16]. In this paper, the most commonest HPV types in the general population were types $16,31,52,68$ and 51 .
Four years ago, de Sanjosé et al. [17] published data about the prevalence of HPV infection in female residents in an area of Barcelona (Spain). In this population, the ageadjusted HPV prevalence was $3 \%$ and the most common types of HPV infection were type 16, 35 and 31 .

Recently, another study has been performed in Spain in women with abnormal cervical cytology [18]. In this manuscript, the highest percentage of women with a positive result for HPV was found in patients with HSIL.

Other Spanish studies have been focused in some subpopulations such as migrant women from Latin America [19] and in imprisoned women [20].

In Andalusia, a southern Spain region, until this moment there are not previously published data about prevalence and HPV types distribution, so further evaluation on this issue needs to be addressed.

The main problem of these studies is their heterogeneicity taking into account the different methods used to evaluate the HPV infections. Until now, the hybrid capture method (Digene ${ }^{\circledR}$ HPV test) is the only FDA

Table 5. Correlation Between Histological Diagnosis and HPV DNA Detection for Cervical Samples. Granada, Spain

\begin{tabular}{|c|c|c|c|c|c|}
\hline \multirow{2}{*}{ Biopsy Diagnosis } & \multicolumn{5}{|c|}{ HPV } \\
\cline { 2 - 6 } & Negative & Low-Risk & High-Risk & Probable High-Risk & Multiple Infections \\
\hline \hline Inflammatory lesions & 19 & 14 & 16 & 12 & 6 \\
\hline ASCUS (Atypia borderline) & 5 & 0 & 4 & 2 & 11 \\
\hline LSIL & 4 & 13 & 26 & 0 & 14 \\
\hline HSIL & 0 & 4 & 9 & 3 & 7 \\
\hline No histological study & 26 & 7 & & 3 \\
\hline
\end{tabular}

* In 3 cases DNA was not obtained. 
approved test to screen for the presence or absence of high/low-risk oncogenic HPV types [21]. However, several other technologies are being used for this purpose such as the in situ hybridization [22], polymerase chain reaction [23], real-time PCR [14] and new array methods [13]. It is apparent that there are substantial differences with respect to the sensitivity of the different diagnostic methods that should be assessed. In general in our study, the concordance with respect to the results obtained from both institutions was high, but some differences could be observed; real-time PCR (Roche) seems more sensitive than array method (Genomica). For example, in one case in which we did not found DNA, the use of a real-time PCR method in this sample led to obtain a positive result (three HPV types). For this reason, we believe that the performance of some comparative studies of the different methods from detection of HPV would be advisable in a high population of patients and with the most homogeneous conditions possible.

In our study there are several data that could be enhanced. Firstly, a higher percentage of infection with multiple types was obtained (33 samples/18.9\%) with respect to other Spanish studies $[10,17]$, and the majority of them were caused with two HPV types. In the majority of articles HPV types 16, 18, 31 were the most common HPV types obtained [3, 15, 17]. However, in our study HPV type 18 only was obtained in two samples $(1.1 \%)$. These data are agreeing with other published studies in Spain $[17,18]$, in which HPV type 18 does not appear as a common type in the general population in our country. Secondly, HPV type 16 was the most common type in all studies, but there was some differences with respect to the prevalence to the other HPV types. In our study, the prevalence of HPV type 16 was lower than those described in the medical literature (about $50 \%$ ) [15]. HPV type 16 and 18 are the two main target of both vaccines, so the address of prevalence of these types is important. In the majority of studies, HPV 16 and 18 cause $70-80 \%$ of lesions $[3,15,17]$; however, in our study the prevalence of both types taken together was $22.3 \%$. This fact is highlighted for the introduction of vaccines in a precise population. From an epidemiological point of view, the performance of these kind of works in a local level is important, because it is necessary to know the different epidemiological patterns to apply the correct diagnostic, prophylactic and therapeutically measures in each area.

Third, in the majority of women included in our study, the HPV infections (high and low-risk types) disappeared promptly in the follow-up (6-12 months), so it could be speculate that the strength control of these patients is the better attitude and no additional treatment is necessary for them. The high percentage of infection with probable highrisk types (28 samples/16.09\%) is another factor to take into account in order to keeping alert with these women.

Fourth, another important result is the low correlation between the histological lesions and the HPV DNA detection for cervical samples (Table 5). In our opinion, this fact could be due to several reasons: the presence of high variability in harvesting samples (no clearly visible or established lesions, endocervical location, suboptimal collection of samples), the different sensitivity and specificity of our diagnosis method (Table 2) with respect to other methods, the biological characteristics of the viruses and finally the variability in the interpretation of the histological features and absence of reliable criteria. This feature is an important issue because the histological diagnosis in cervical pathology is the "gold standard". The absence of complete reliability is a common problem in all kinds of biopsies come from different organs and tissues, above all in low-grade neoplastic lesions. Moreover, in spite of this inconvenient, microscopic evaluation of cervical biopsies has a high level of accuracy done by expert pathologists. Thus, the absence of concordance between HPV typing and the histological diagnosis means that HPV could be present in cervical epithelial cells without produce atypical changes or minimal cellular alterations identified by means of cytology. In fact, in our study 48 of 67 (71.6\%) samples with reactive conditions were positive for HPV infection, although 9 of 14 (64.2\%), 64 of $68(94.1 \%)$ and 24 of $24(100 \%)$ were also positive for HPV DNA and were diagnosed of atypia borderline, LSIL and HSIL respectively. In addition, another relevant aspect is the presence of 26 of $52(50 \%)$ cases with HPV infection in patients without changes in colposcopic examination (no biopsies were taken by the gynaecologist).

This fact is important in order to avoid that healthy women without dysplasic atypical changes in the cervical epithelium (histopathological study) could be considerate ill, only based in positive HPV typing. Evidence-based information must be released to the general population for public health educational purposes.

Quality controlled screening programs and preventive measures are really necessaries to reduce the incidence of HPV infection and the incidence of cervical cancer. Epidemiologic studies clearly demonstrated that infection with HPV high-risk types is essential for the development of cervical cancer [24]. At the moment, the only two prophylactic vaccines available for clinical use are generated by recombinant technologies and are composed of viral-like particles. Cervarix produced by GlaxoSmithKline is a bivalent vaccine (HPV 16-18), while Gardasil produced by Merck is a quadrivalent vaccine (HPV 6-11-16-18). Until now, little is known about their comparative efficacy, safety and duration of protection. These data will be available in the future when these vaccines be widely worldwide applied.

However, after the analysis of the different epidemiological published studies, it can see that the epidemiological patterns of HPV types prevalence could be different depending of the region of origin. In many countries, these vaccines could prevent $50 \%$ of all HPV infections in women without cytological alterations [3], but also $70-80 \%$ of HPV infections caused in high-risk lesions or invasive carcinoma [15]. In our area, and taking into account the data shown in this paper, only approximately $30 \%$ of HPV infections that led to precursor neoplastic cervical lesions could be favoured with the introduction of the recently approved vaccines. In addition, it is necessary to study the prevalence of HPV types related to cervical carcinoma because we do not know the prevalence of HPV types that cause invasive carcinoma in our geographic area (the types that cause invasive cancer have a different distribution with respect those causing preneoplastic lesions) [15]. Thus, assessment of the distribution of HPV types in each health area will be a very important factor to indicate the vaccines. They imply that more epidemiological studies 
are necessary to know the HPV type prevalence in each area and regional variation in the distribution of certain HPV types should be taken into account in the future production of vaccines.

Moreover, it can speculate that the generalized introduction of these vaccines could produce an epidemiological pattern change, so in the further several years the majority of HPV infections will be produced for different HPV types with oncogenic risk than 16 and 18. Other situations that could be considerate are as follows: HPV prevalence in immunodepressed patients and the effect of vaccine in the origin and evolution of non-gynaecological HPV-induced cancers (oral, anal, oropharyngeal).

\section{CONCLUSION}

Regional variations in prevalence and distribution of HPV types have been demonstrated in several epidemiological studies. In some countries such as Spain, these changes could also be due to the increase of migrant women from Latin America [19], Eastern Europe [25] and North Africa [26] countries, so health service providers must establish continuous localized screening programs in order to identify the HPV infections. Moreover, apart of the change of the HPV types distribution pattern, an increase of the multiple infections can be observed. In our study, a higher percentage of infection with multiple types was obtained (33 samples/18.9\%) with respect to other Spanish studies [10, 17]. Also, in this study, the prevalence of HPV type 16 was lower than those described in the medical literature (about $50 \%$ ) [15]. In the majority of women included in our study, HPV infections (high and low-risk types) disappeared promptly in the follow-up (6-12 months) and there was low correlation between the histological lesions and the HPV DNA detection for cervical samples, so further studies are neccesaries to address this fact.

\section{ACKNOWLEDGEMENT}

We would like acknowledge Ms. Angela Barnie for checking the English language of this manuscript.

\section{REFERENCES}

[1] Baker TS, Newcomb WM, Olson NH, Cowsert LM, Olson C, Brown JC. Structures of bovine and human papillomaviruses. Analysis by cryoelectron microscopy and three-dimensional image reconstruction. Biophysics 1991; 60: 1445-56.

[2] De Villiers EM, Fauquet C, Broker TR, Bernard HU, zur Hausen H. Classification of papillomavirus. Virology 2004; 324: 17-27.

[3] De Sanjosé S, Díaz M, Castellsagué X, et al. Worldwide prevalence and genotype distribution of cervical human papillomavirus DNA in women with normal cytology: a metaanalysis. Lancet Infect Dis 2007; 7: 453-9.

[4] Burd EM. Human papillomavirus and cervical cancer. Clin Microbiol Rev 2003; 16: 1-17.

[5] Muñoz N, Bosch FX, de Sanjosé S, et al. The causal link between human papillomavirus and invasive cervical cancer: a population-based case-control study in Colombia and Spain. Int J Cancer 1992; 52: 7439.
[6] Walboomers JM, Jacobs MV, Manos MM, et al. Human papillomavirus is a necessary cause of invasive cervical cancer worldwide. J Pathol 1999; 189: 9-12.

[7] Bosch FX, Lovincz A, Muñoz N, Meijer CJ, Shah KV. The causal relation between human papillomavirus and cervical cancer. J Clin Pathol 2002; 55: 244-65.

[8] Parkin DM. The global health burden of infection-associated cancers in the year 2002. Int J Cancer 2006; 118: 3030-44.

[9] Pérez-Ayala M, Ruiz-Cabello F, Esteban F, et al. Presence of HPV 16 sequences in laryngeal carcinomas. Int J Cancer 1990; 46: 8-11.

[10] Muñoz N, Bosch FX, de Sanjosé S, et al. Epidemiologic classification of Human papillomavirus types associated with cervical cancer. N Engl J Med 2003; 348: 518-27.

[11] Papanicolaou GN. A survey of actualities and potentialities of exfoliative cytology in cancer diagnosis. Ann Intern Med 1949; 31: 661-74.

[12] Bosch FX, Cuzick J, Schiller J, et al. HPV vaccines and screening in the prevention of cervical cancer. Vaccine 2006; Suppl 3: S1-S264

[13] Chacón J, Sanz I, Rubio MD, et al. Detection and genotyping of highrisk human papillomavirus in cervical specimens. Enf Infecc Microbiol Clin 2007; 25: 311-6.

[14] Coutlee F, Rouleau D, Ghattas G, et al. Confirmatory real-time PCR assay for HPV-52 infection in anogenital specimens screened for HPV infection with the LINEAR ARRAY HPV genotyping test. J Clin Microbiol 2007; 45: 3821-3.

[15] Zuna RE, Allen RA, Moore WE, Lu Y, Mattu R, Dunn ST. Distribution of HPV genotypes in 282 women with cervical lesions: evidence for three categories of intraepithelial lesions based on morphology and HPV type. Mod Pathol 2007; 20: 167-74.

[16] Ortiz M, Torres M, Muñoz L, et al. Oncogenic human papillomavirus (HPV) type distribution and HPV type 16 E6 variants in two Spanish population groups with different levels of HPV infection risk. J Clin Microbiol 2006; 44: 1428-34.

[17] De Sanjosé S, Almirall R, Lloveras B, et al. Cervical human papillomavirus infection in the female population in Barcelona, Spain. Sex Transm Dis 2003; 30: 788-93

[18] González-Bosquet E, Almagro MM, Mora I, Suñol M, Callejo J, Lailla JM. Prevalence of human papilloma virus infection of the uterine cervix in women with abnormal cervical cytology. Eur J Gynaecol Oncol 2006; 27: 135-8.

[19] González C, Ortiz M, Canals J, et al. Higher prevalence of human papillomavirus infection in migrant women from Latin America in Spain. Sex Transm Infect 2006; 82: 260-2.

[20] González C, Canals J, Ortiz M, et al. Prevalence and determinants of high-risk human papillomavirus (HPV) infection and cervical cytological abnormalities in imprisoned women. Epidemiol Infect 2008; 136: 215-21.

[21] Department of Health and Human Services. Food and Drug Administration. Digene Hybrid Capture $2\left(\mathrm{HC}_{2}\right)$ High-risk HPV DNA test. Premarket Approval Application. P890064(S9)/2004M-0147. Rockville, MD. March 31, 2003.

[22] Huang CC, Qiu JT, Kashima ML, Kurman RJ, Wu TC. Generation of type-specific probes for the detection of single-copy human papillomavirus by a novel in situ hybridization method. Mod Pathol 1998; 11: 971-7.

[23] Kleter B, Van Doorn LJ, Schrauwen L, et al. Development and clinical evaluation of a highly sensitive PCR-reverse hybridization line probe assay for detection and identification of anogenital human papillomavirus. J Clin Microbiol 1999; 37: 2508-17.

[24] Bosch FX, de Sanjosé S. Human papillomavirus and cervical cancerburden and assessment of causality. J Natl Cancer Inst Monogr 2003; 31:3-13.

[25] Kulmala SM, Shabalova IP, Petrovitchev N, Syrjänen KJ, Gyllensten UB, Syrjänen SM. Prevalence of the most common high-risk HPV genotypes among women in three new independent states of the former Soviet Union. J Med Virol 2007; 79: 771-81

[26] Hassen E, Chaieb A, Letaief M, et al. Cervical human papillomavirus infection in Tunisian women. Infection 2003; 31: 143-8.

This is an open access article licensed under the terms of the Creative Commons Attribution Non-Commercial License (http: //creativecommons.org/licenses/by-nc/ 3.0/) which permits unrestricted, non-commercial use, distribution and reproduction in any medium, provided the work is properly cited. 